\title{
Development of Steering Laws to Assist the Driving of a Independent Front and Rear Steering Vehicle
}

\author{
Louis DAMBACHER ${ }^{1}$, Benjamin BOUDON ${ }^{1}$, Nicolas BOUTON ${ }^{1}$, ${\text { Roberto } \text { LOT }^{2} \text {, Nicolas }}^{\text {, }}$ \\ LALANDE $^{3}$, Roland LENAIN ${ }^{4}$
}

${ }^{1}$ Université Clermont Auvergne,CNRS SIGMA Clermont, Institut Pascal 63000 Clermont-Ferrand, France [louis.dambacher, benjamin.boudon, nicolas.bouton]@sigma-clermont.fr

\author{
${ }^{3}$ Airbus Helicopters \\ Aéroport de Marseille Provence \\ 13700 Marignane, France \\ nicolas.lalande.as@gmail.com
}

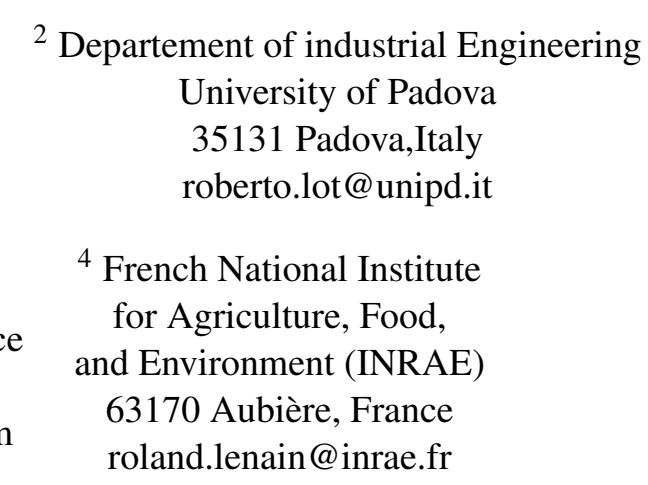

\begin{abstract}
This paper proposes a control strategy to control the yaw rate of a 2-independentsteering-gears vehicle operated by a driver. The approach is based on adaptive techniques to account the slip effects. An extended kinematic model is used which takes into account slipping effects through side-slip angles. A 3-steps state observer is proposed to estimate a characteristic parameter defining the tyre-ground contact. This state observer use only the value of control variables and the yaw rate measurement of the vehicle. Thanks to the proposed approach the driver can safely steer the 2steering-gears off-road vehicle using one steering actuator without losing control at high speeds as will be the case with two independent steering actuators.
\end{abstract}

Keywords: 2-steering-gears vehicle, Vehicle dynamics, Yaw rate control, Adaptive control, State observer.

\section{INTRODUCTION}

The purpose of this paper is in the field of assisted ground vehicle dynamics, and more specifically in the development of steering systems for two-steering-gears vehicles. This sector is in regain of interest with the improvement of the on-board electronics. This improvement is highlighted in the automotive field by the optimisation of comfort, agility and driving safety. On-board electronics allows, when the vehicle is equipped, to handle 2 steering gears on one vehicle. Vehicles with two steering gears offer many opportunities to improve the driver's driving comfort:

- Vehicle dynamics are improved, especially on slippery ground.

- Complex manoeuvres that would be impossible to perform with a traditional single-steer vehicle are now possible. Vehicle agility is improved.

- Behaviours that could endanger the driver can be avoided by the intervention of a second steering gear. The stability of the vehicle is improved.

- From an energy point of view, the presence of two steering gears makes it possible to "distribute" the wear of the front and rear tyres. The life of the tyres is therefore increased.

In short, the presence of two independent steering gears allows the driver to have better control of the vehicle, and human/vehicle interaction is therefore optimised. 
However, despite all these advantages, the use of a vehicle with two independent steering gears can also be very dangerous for the driver. Indeed, without assistance, a vehicle of this type id difficult to handle, especially at high speed. It is to answer this problem that it was chosen to develop the two following contributions in this paper:

- The development of a control law that allows the driver to drive a 2-steering-gears vehicle in off-road conditions without putting himself in danger.

- The development of a state observer reflecting the behaviour of the tyre-ground contact, which is essential for the implementation of an efficient control law. The constraint of this observation is that it must be done at the lowest cost and therefore use the fewest possible sensors.

For this purpose, it was chosen to use a model adapted to the control, but still sufficiently sophisticated to take into account the main phenomena associated to the dynamics of the vehicle and the interactions of the tyres with the ground. Indeed, in previous works, very advanced dynamic representations can be found [1]. But the large number of parameters makes them difficult to use for control. In the opposite case, simple kinematic models have been developed for control purposes [2]. However, these models are not representative of various phenomena, in particular: the lack of grip, the vehicle inertia, or the load transfer phenomenon. In this paper a hybrid approach is used allowing the use of simplified models adapted in real time by observers [3], [4]. These observers are based on a simplified model of tyre/ground contact extending the traditional definition [5] of the cornering phenomenon.

\section{Vehicle modelling for control purposes}

The vehicle model used to test the various control laws developed is a very simplified model of the vehicle. The vehicle is reduced to a bicycle shape (figure 1) where the front (and the rear axle) is considered as a single wheel. Nevertheless this modelling takes into account the phenomena of slipping, by introducing the presence of side-slip angle, at the front, at the rear and at the G point of the model. This model is called "Extended Kinematic Model" [6],[7]. 


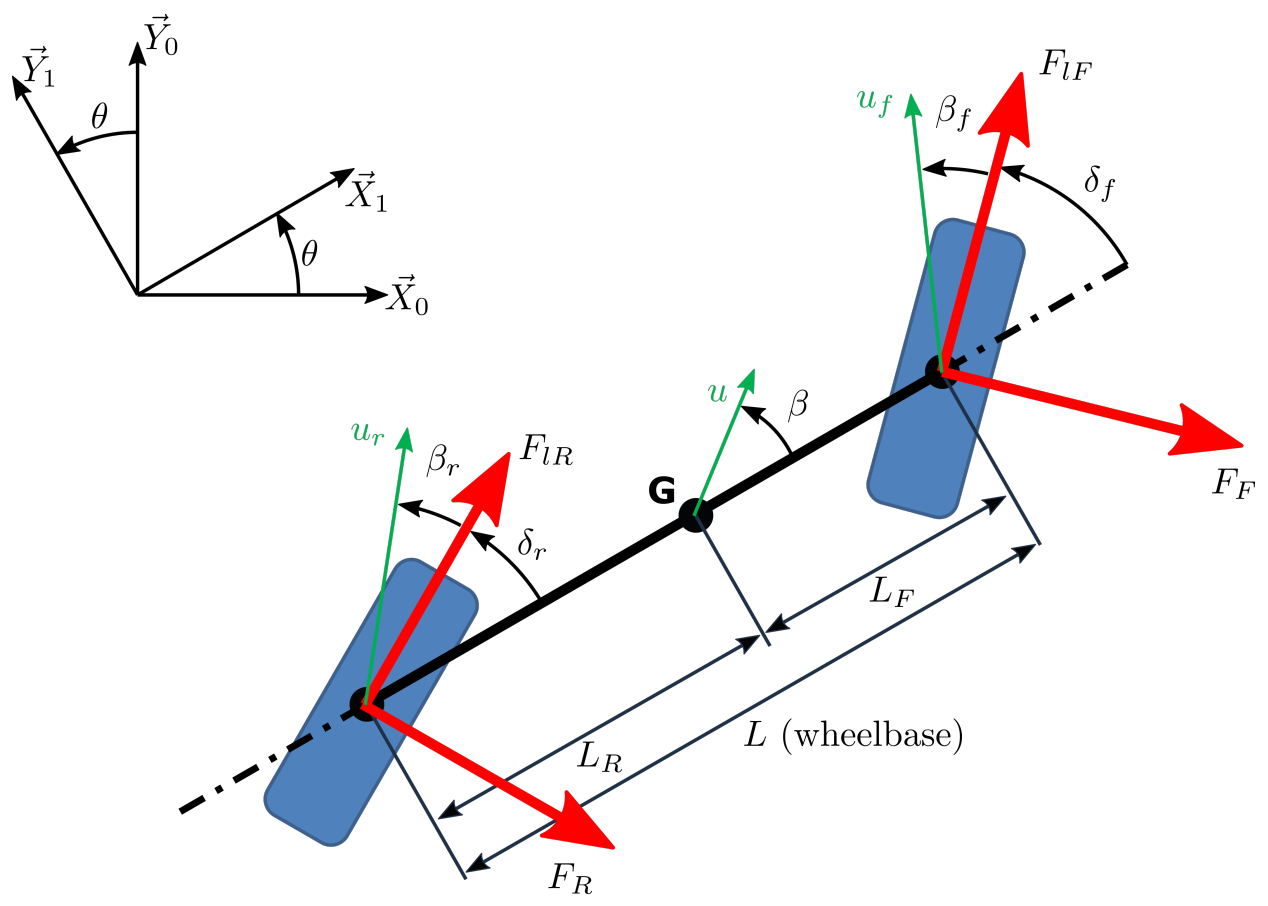

Figure 1. 4WS extended kinematic model

The notations used are as follows:

$\theta:$ Yaw angle

$\delta_{r}$ : Rear steering angle

$\delta_{f}$ : Front steering angle

$u_{r}:$ Speed at the center of the rear gear

$u_{f}:$ Speed at the center of the front gear

$u$ : Speed at vehicle G-point

$\beta_{r}$ : Rear slip angle

$\beta_{f}$ : Front slip angle

$\beta$ : Global slip angle at G-point

$F_{l R}:$ Rear longitudinal contact force

$F_{l F}:$ Front longitudinal contact force

$F_{R}$ : Rear lateral contact force

$F_{F}:$ Front lateral contact force

$L:$ Wheelbase of the vehicle

\subsection{Kinematic equations}

A first kinematic analysis allows us to establish the link between the speed of the rear axle $u_{r}$ and the speed at the G point $u$ as well as the link between the rear side-slip angle $\beta_{r}$ and the global side-slip angle $\beta$.

The chassis is considered as a rigid body. The kinematic relation (1) inside the body can be written: 


$$
\begin{aligned}
& \vec{u}_{r}=\vec{u}+\left(L_{R} \vec{X}_{1}\right) \wedge \dot{\theta} \vec{Z}_{1} \\
&=\vec{u}-L_{R} \dot{\theta} \vec{Y}_{1} \\
& \Rightarrow\left(\begin{array}{c}
u_{r} \cos \left(\delta_{r}+\beta_{r}\right) \\
u_{r} \sin \left(\delta_{r}+\beta_{r}\right) \\
0
\end{array}\right)_{1}=\left(\begin{array}{c}
u \cos (\beta) \\
u \sin (\beta) \\
0
\end{array}\right)_{1}-\left(\begin{array}{c}
0 \\
L_{r} \dot{\theta} \\
0
\end{array}\right)
\end{aligned}
$$

The projections on the axis $\vec{X}_{1}$ and $\vec{Y}_{1}$ enable to obtain 2 scalar equations :

$$
\begin{gathered}
\Leftrightarrow\left\{\begin{array}{c}
u_{r} \cos \left(\delta_{r}+\beta_{r}\right)=u \cos (\beta) \\
u_{r} \sin \left(\delta_{r}+\beta_{r}\right)=u \sin (\beta)-L_{R} \dot{\theta}
\end{array}\right. \\
\Rightarrow\left\{\begin{array}{c}
u=\frac{u_{r} \cos \left(\delta_{r}+\beta_{r}\right)}{\cos (\beta)} \\
\tan \left(\delta_{r}+\beta_{r}\right)=\tan (\beta)-\frac{L_{2} \dot{\theta}}{u \cos (\beta)}
\end{array}\right. \\
\Rightarrow\left\{\begin{array}{c}
u=\frac{u_{r} \cos \left(\delta_{r}+\beta_{r}\right)}{\cos (\beta)} \\
\beta_{r}=\arctan \left(\tan (\beta)-\frac{L_{R} \dot{\theta}}{u \cos (\beta)}\right)-\delta_{r}
\end{array}\right.
\end{gathered}
$$

The equations linking the speed of the front gear $u_{f}$ and the speed at the G point $u$ as well as the relation between the front side-slip angle $\beta_{f}$ and the global side-slip angle $\beta$ are obtained in the same way. The relations are as follows:

$$
\Rightarrow\left\{\begin{array}{c}
u=\frac{u_{f} \cos \left(\delta_{f}+\beta_{f}\right)}{\cos (\beta)} \\
\beta_{f}=\arctan \left(\tan (\beta)+\frac{L_{F} \dot{\theta}}{u \cos (\beta)}\right)-\delta_{f}
\end{array}\right.
$$

\subsection{Tyre force model}

Due to the application of a lateral force on the wheel, during its own rotation, the tyre/ground contact area moves from the position $C_{0}$ to a position $C$. The angle $\beta$ formed by the straight line $\left(C_{0} C\right)$ and the longitudinal axis in the main wheel plane is called the side-slip angle.

The most well-known way of modelling tyre-ground contact is Pacejka's so-called Magic Formula [8]. This formula links, among other things, the lateral force $\mathrm{Y}$ and the side-slip angle $\beta$. However, this model is not suitable for an off-road application. Moreover, because of the numerous parameters involved in this formula, it is not adapted to the control. It is chosen here to model the tyre-ground contact using an adaptable cornering stiffness $C_{e}$ as follows:

$$
Y=C_{e} \beta
$$

The adaptability of the value of the cornering stiffness $C_{e}$ is done thanks to the "Observer" block detailed in the paragraph 4 . The general principle is illustrated in the figure 2 


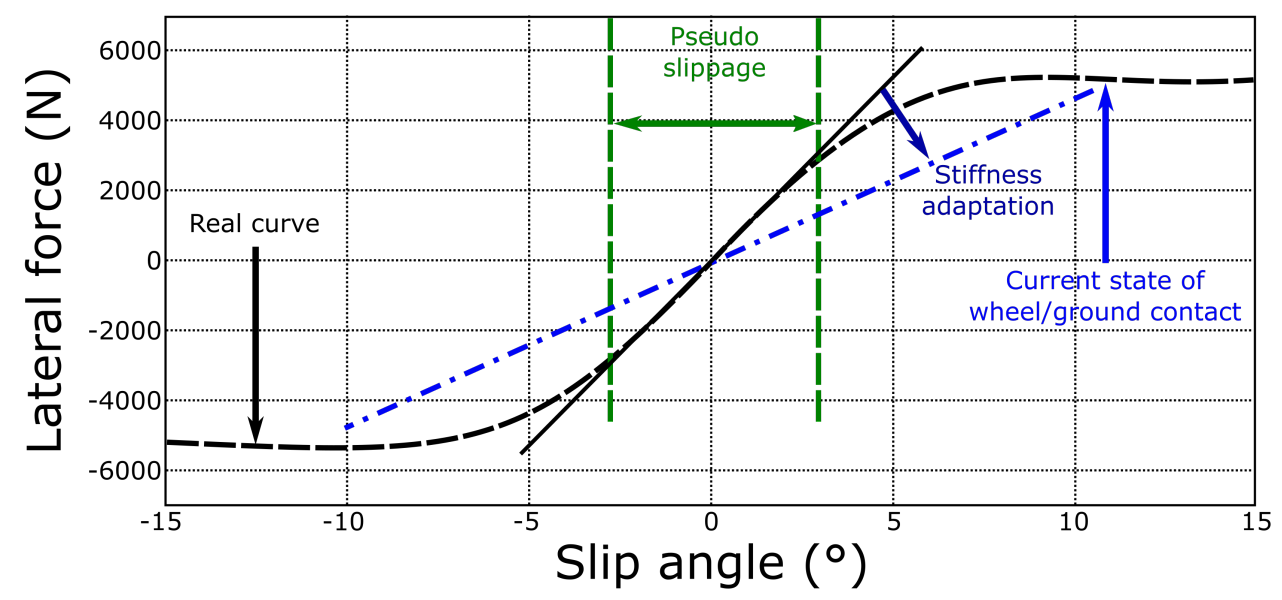

Figure 2. Principe of cornering stiffness $C_{e}$ adaptability

As shown in the figure 2, a linear relation (black line) with $C_{e}$ constant is valid only for small side-slip angles. To describe the real curve (black dashed line) for higher side-slip angles the slope of the line, which is $C_{e}$, is modified in real time, this is the adaptation of the cornering stiffness (blue dashed line).

\subsection{Dynamic equations}

The Physical phenomena and the modelling assumptions are as follows :

- The wheel/ground contact forces are modelled as explained in subsection 2.2 .

- Only one rigid solid is considered here, that of the vehicle's chassis.

- Only one inertia is taken into account, that of the chassis in the yaw plane. The other inertia, such as those of the wheels for example, are neglected.

From Newton's second law, it is possible to determine the system of equations (9) :

$$
\left\{\begin{array}{c}
\dot{\beta}=\frac{1}{u m}\left[F_{l f} \sin \left(\delta_{f}-\beta\right)+F_{l r} \sin \left(\delta_{r}-\beta\right)-C_{f} \beta_{f} \cos \left(\delta_{f}-\beta\right)-C_{r} \beta_{r} \cos \left(\delta_{r}-\beta\right)\right]-\dot{\theta} \\
\ddot{\theta}=\frac{1}{I_{z}}\left[L_{r} C_{r} \beta_{r} \cos \left(\delta_{r}\right)-F_{l r} L_{r} \sin \left(\delta_{r}\right)-L_{f} C_{f} \beta_{f} \cos \left(\delta_{f}\right)+F_{l f} L_{f} \sin \left(\delta_{f}\right)\right]
\end{array}\right.
$$

This is a system of ordinary differential equations (ODEs) with two inputs : $\delta_{f}$ and $\delta_{r}$ describing the evolution of the global side-slip angle $\beta$ and the evolution of the yaw rate of the vehicle $\dot{\theta}$.

To fully describe the behaviour of the vehicle in the yaw plane, three kinematic equations must be added to the equations of system (9). This is the system of equations (10) :

$$
\left\{\begin{array}{l}
\dot{\beta}=\frac{1}{u m}\left[F_{l f} \sin \left(\delta_{f}-\beta\right)+F_{l r} \sin \left(\delta_{r}-\beta\right)-C_{f} \beta_{f} \cos \left(\delta_{f}-\beta\right)-C_{r} \beta_{r} \cos \left(\delta_{r}-\beta\right)\right]-\dot{\theta} \\
\ddot{\theta}=\frac{1}{I_{z}}\left[L_{r} C_{r} \beta_{r} \cos \left(\delta_{r}\right)-F_{l r} L_{r} \sin \left(\delta_{r}\right)-L_{f} C_{f} \beta_{f} \cos \left(\delta_{f}\right)+F_{l f} L_{f} \sin \left(\delta_{f}\right)\right] \\
u=\frac{u_{r} \cos \left(\beta_{r}+\delta_{r}\right)}{\cos (\beta)} \\
\beta_{r}=\arctan \left(\tan (\beta)-\frac{L_{r} \dot{\theta}}{u \cos (\beta)}\right)-\delta_{r} \\
\beta_{f}=\arctan \left(\tan (\beta)+\frac{L_{f} \dot{\theta}}{u \cos (\beta)}\right)-\delta_{f}
\end{array}\right.
$$

It is the equation linking the speed of the rear axle $u_{r}$ and the speed at the G point $u$ that is implemented, as it is the rear wheels that are driven. 


\section{Steering law}

The steering law developed is designed to assist the driver in driving the two-steering-gears vehicle at high speed. This steering law controls the rear axle while the front axle is steered by the driver. The main objective of this steering law is to limit the effects of slippery ground on the driver's driving and to make the driver feel as if he or she is driving a traditional vehicle with one steering gear while driving on difficult ground in a vehicle with two steering gears.

As can be seen on the figure 3, the control architecture is composed of two main blocks: the control loop and the observer, which will be detailed in the section 4. From measurements made at the output of the vehicle model, the observer aims to reconstruct variables that feed the control law.

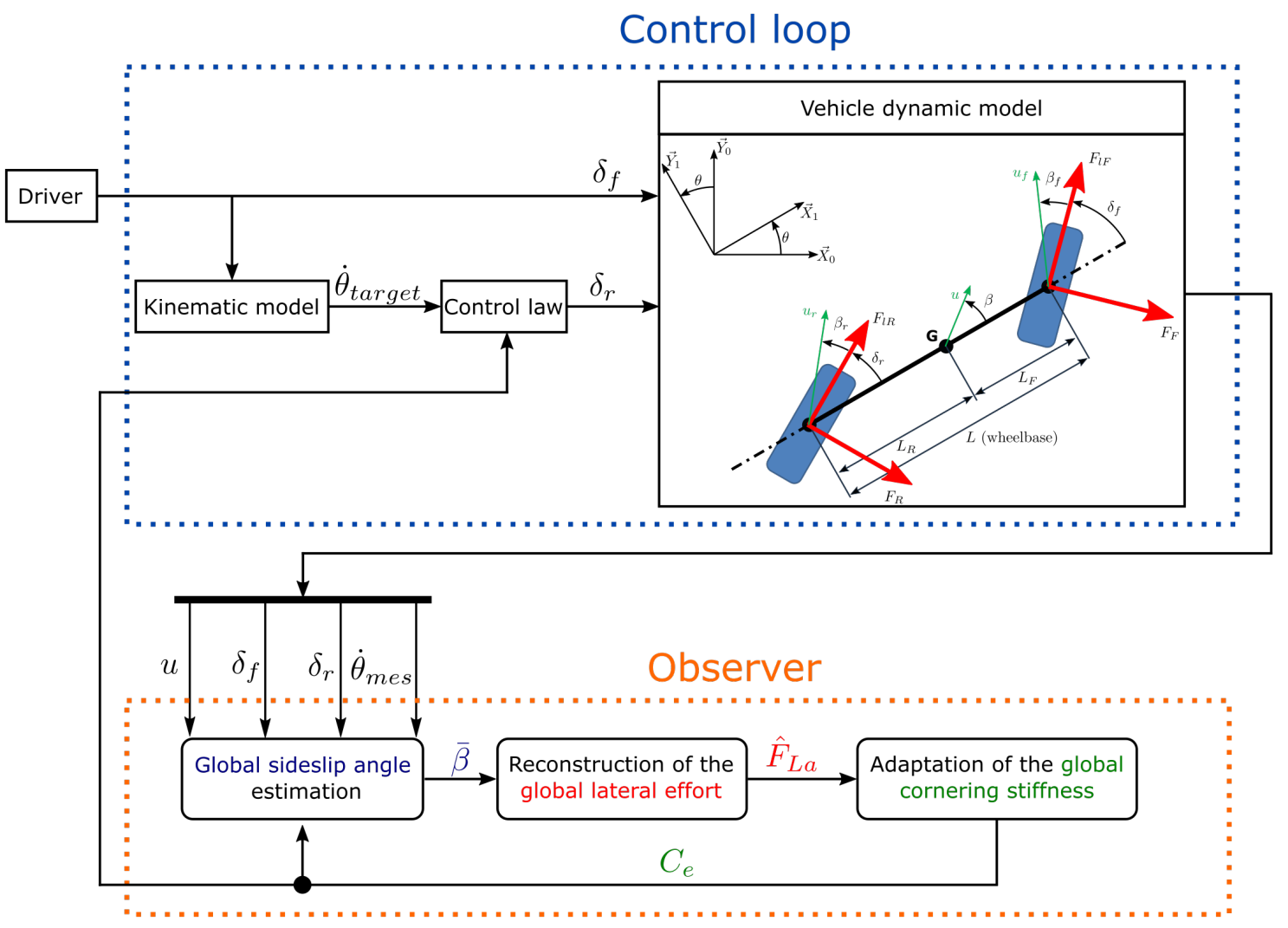

Figure 3. Global architecture of the control system

\subsection{Principle of Yaw Rate Control}

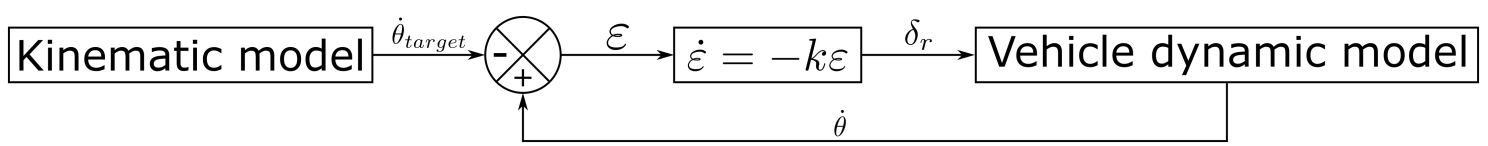

Figure 4. Yaw rate control

The target yaw rate $\dot{\theta}_{\text {target }}$ is obtained from a kinematic model of a bicycle shape vehicle (like in figure 1) modelled without slippage. More precisely, the contact between the wheels and the ground is considered as a rolling without slipping (RWS) contact. A purely geometrical analysis allows to define the result of equation (11), defining the yaw rate of the vehicle in rolling without slipping conditions. For a vehicle, steered by the front gear, the target yaw rate can be obtained with the following relation :

$$
\dot{\theta}_{\text {target }}=\frac{u_{r} \tan \left(\delta_{f}\right)}{L}
$$




\subsection{Implementation of the control law}

The error $\varepsilon$ is defined as follows:

$$
\varepsilon=\dot{\theta}-\dot{\theta}_{\text {target }}
$$

The control law is based on an exponential decrease of the error to 0 . This translates mathematically into the differential equation (13):

$$
\begin{aligned}
\dot{\varepsilon} & =-k \varepsilon \text { with } k>0 \\
\Leftrightarrow \ddot{\theta} & =k\left(\dot{\theta}_{\text {target }}-\dot{\theta}\right)
\end{aligned}
$$

From (9) and neglecting the longitudinal forces, the following equation can be written :

$$
\ddot{\theta}=\frac{1}{I_{z}}[\overbrace{L_{r} C_{r}}^{A_{r}} \beta_{r} \cos \left(\hat{\delta}_{r}\right)-\overbrace{L_{f} C_{f}}^{A_{f}} \beta_{f} \cos \left(\delta_{f}\right)]
$$

The aim is to isolate the rear steering angle $\delta_{r}$ variable. Since $\beta_{r}$ depends on $\delta_{r}$, it is not possible to isolate it easily. A back-stepping strategy is used here, using the intermediate control variable $u_{\beta_{r}}$, the target value for $\beta_{r}$. This is illustrated in Figure 5 .

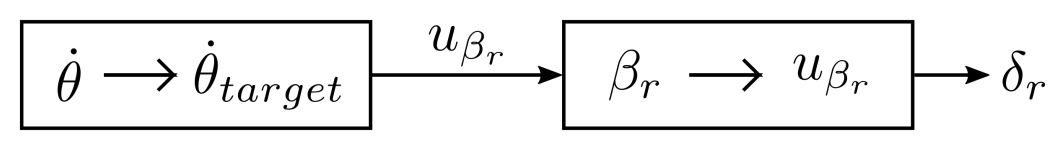

Figure 5. Back-stepping Strategy

By replacing the expression (15) of $\ddot{\theta}$ in (14), it is possible to easily isolate $\beta_{r}$ and thus determine the value $u_{\beta_{r}}$ :

$$
u_{\beta_{r}}=\frac{1}{A_{r} \cos \left(\hat{\delta}_{r}\right)}\left[I_{z} k\left(\dot{\theta}_{\text {target }}-\dot{\theta}\right)+A_{f} \beta_{f} \cos \left(\delta_{f}\right)\right]
$$

$\beta_{r}$ and $\delta_{r}$ are kinematically related according to equation (17):

$$
\beta_{r}=\overbrace{\arctan \left(\tan (\beta)-\frac{L_{f} \dot{\theta}}{u \cos (\beta)}\right)}^{B_{r}}-\delta_{r}
$$

And finally the the expression for $\delta_{r}$ can be determined :

$$
\delta_{r}=B_{r}-u_{\beta_{r}}
$$

\section{Cornering Stiffness observer}

\subsection{State observer}

The purpose of an observer is to estimate an unmeasurable state $\hat{X}$, from measurable variables $Y$ and control variables $v$ in order to be able to use them in the control law. Figure 6 illustrates how a state observer is used in control. 


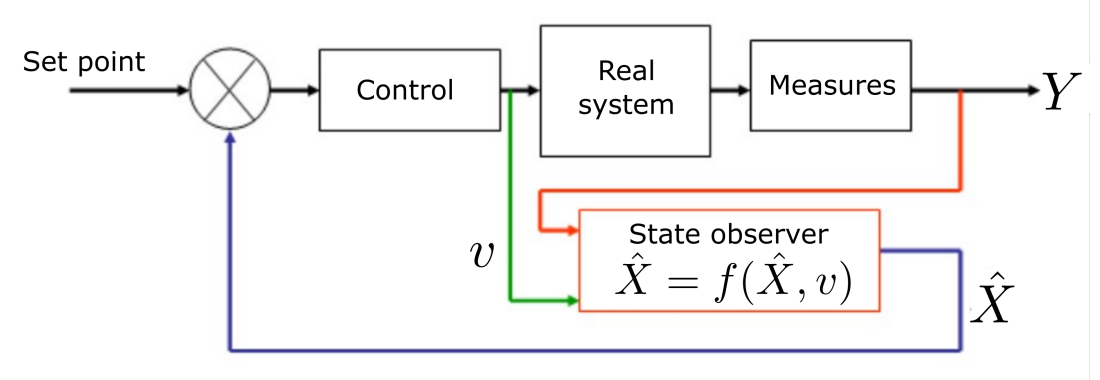

Figure 6. State observer

\subsection{Cornering stiffness observation}

As mentioned in paragraph 2.2, the contact between the tyres and the ground is modelled using a linear relation between the lateral force $Y$ and the side-slip angle $\beta$. The relation is in fact nonlinear, so it was chosen to adapt this coefficient $C_{e}$ from this relation. The coefficient $C_{e}$ is called the cornering stiffness, so this is the cornering stiffness adaptation strategy. This strategy, shown in Figure 7, is composed of 3 steps detailed below. The last step is of course the estimation of the cornering stiffness, which is then fed back into the operations of the first step. The cornering stiffness is therefore regulated.

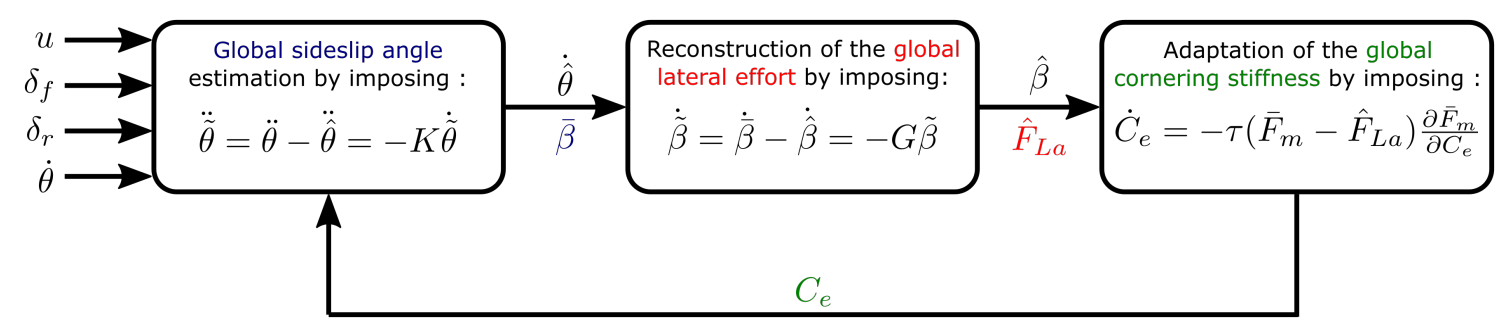

Figure 7. Cornering stiffness observer

The notations used are as follows:

$\hat{X}$ : Estimated variable

$\bar{X}$ : Measured variable or reconstruction of a measurement

$\tilde{X}=\bar{X}-\hat{X}$

Step 1:

Here, the aim is to reconstruct the global side-slip angle $\bar{\beta}$ from the measurement (by sensor) of the yaw rate $\dot{\theta}$. The strategy adopted here is to make converge an estimation $\dot{\hat{\theta}}$ of the yaw rate to the yaw rate $\dot{\theta}$ measured by sensor, using $\bar{\beta}$ considered here as a control variable. The method for convergence from $\dot{\hat{\theta}}$ to $\dot{\theta}$ is to impose an exponential decrease of the error, as in paragraph 3.1:

$$
\ddot{\tilde{\theta}}=-k \dot{\tilde{\theta}} \Leftrightarrow \ddot{\theta}-\ddot{\hat{\theta}}=-k(\dot{\theta}-\dot{\hat{\theta}}) \text { with } k>0
$$

The side-slip angle $\bar{\beta}$ appears in the expression of $\ddot{\hat{\theta}}$ thanks to the the second equation of (9). However, due to the non-linearity of the last two equations of the system (10), it is very difficult to extract $\bar{\beta}$. It was therefore decided to linearise these equations and not to take into account the longitudinal forces. The simplified expression of $\ddot{\theta}$ is as follows:

$$
\ddot{\hat{\theta}}=a_{11} \dot{\hat{\theta}}+a_{12} \bar{\beta}+b_{11} \delta_{f}+b_{12} \delta_{r}
$$

With :

$$
\begin{array}{ll}
a_{11}=-\frac{C_{e}\left(L_{f}^{2} \cos \left(\delta_{f}\right)+L_{r}^{2} \cos \left(\delta_{r}\right)\right)}{u I_{z}} & a_{12}=\frac{C_{e}\left(L_{r} \cos \left(\delta_{r}\right)-L_{f} \cos \left(\delta_{f}\right)\right)}{I_{z}} \\
b_{11}=\frac{C_{e} L_{f} \cos \left(\delta_{f}\right)}{I_{z}} & b_{12}=-\frac{C_{e} L_{r} \cos \left(\delta_{r}\right)}{I_{z}}
\end{array}
$$


By replacing this in equation (19) :

$$
\begin{aligned}
\ddot{\theta}-\ddot{\hat{\theta}} & =-k \dot{\tilde{\theta}} \\
\ddot{\theta}-a_{11} \dot{\hat{\theta}}-a_{12} \bar{\beta}-b_{11} \delta_{f}-b_{12} \delta_{r} & =-k \dot{\tilde{\theta}}
\end{aligned}
$$

Finally :

$$
\bar{\beta}=\frac{\ddot{\theta}-a_{11} \dot{\hat{\theta}}-b_{11} \delta_{f}-b_{12} \delta_{r}+k \dot{\tilde{\theta}}}{a_{12}}
$$

Step 2:

Here, the aim is to reconstruct the global lateral force $\hat{F}_{L a}$ from $\bar{\beta}$. The strategy adopted here is to make converge an estimation $\hat{\beta}$ of side-slip angle to $\bar{\beta}$, using $\hat{F}_{L a}$ considered here as a control variable. The method for convergence from $\hat{\beta}$ to $\bar{\beta}$ is to impose an exponential decrease of the error, as in paragraph 3.1 and as the precedent step :

$$
\dot{\tilde{\beta}}=-k \tilde{\beta} \Leftrightarrow \dot{\bar{\beta}}-\dot{\hat{\beta}}=-k(\bar{\beta}-\hat{\beta}) \text { with } k>0
$$

The global lateral force is defined as follows:

$$
\bar{F}_{L a}=C_{e}\left(\beta_{f} \cos \left(\delta_{f}-\bar{\beta}\right)+\beta_{r} \cos \left(\delta_{r}-\bar{\beta}\right)\right)
$$

From (9) and neglecting the longitudinal forces, the following equation can be written :

$$
\dot{\hat{\beta}}=-\frac{1}{u m} \hat{F}_{L a}-\dot{\hat{\theta}}
$$

By replacing this in equation (24) :

$$
\begin{aligned}
\dot{\bar{\beta}}-\dot{\hat{\beta}} & =-k \tilde{\beta} \\
\dot{\bar{\beta}}+\frac{1}{u m} \hat{F}_{L a}+\dot{\hat{\theta}} & =-k \tilde{\beta}
\end{aligned}
$$

Finally :

$$
\hat{F}_{L a}=-u m(\dot{\hat{\theta}}+\dot{\bar{\beta}}+k \tilde{\beta})
$$

Step 3 :

Here, the aim is to reconstruct the global cornering stiffness $C_{e}$. The strategy adopted here is the MIT Rule [9] to make converge $\hat{F}_{L a}$ to $\bar{F}_{L a}$ by adapting the value of $C_{e}$.

The MIT Rule is formulated as follows:

$$
\dot{C}_{e}=-\tau e \frac{\partial e}{\partial C_{e}}
$$

With :

$$
\left\{\begin{array}{l}
e=\bar{F}_{L a}-\hat{F}_{L a} \\
\bar{F}_{L a}=C_{e}\left(\beta_{f} \cos \left(\delta_{f}-\bar{\beta}\right)+\beta_{r} \cos \left(\delta_{r}-\bar{\beta}\right)\right) \\
\hat{F}_{L a}=-u m(\dot{\hat{\theta}}+\dot{\bar{\beta}}+k \tilde{\beta})
\end{array}\right.
$$

Finally by replacing this in equation (30), the following equation can be written :

$$
\dot{C}_{e}=-\tau\left(\bar{F}_{L a}-\hat{F}_{L a}\right)\left(\beta_{f} \cos \left(\delta_{f}-\bar{\beta}\right)+\beta_{r} \cos \left(\delta_{r}-\bar{\beta}\right)\right)
$$

\section{Simulation results}

\subsection{Presentation of the global model}

The Simulink model shown in Figure 8 is designed to simulate the behaviour of the 2-steering-gear vehicle under different simulation scenarios. If tests of different control laws with different vehicle models are to be done, the general architecture of the simulator would be maintained. 


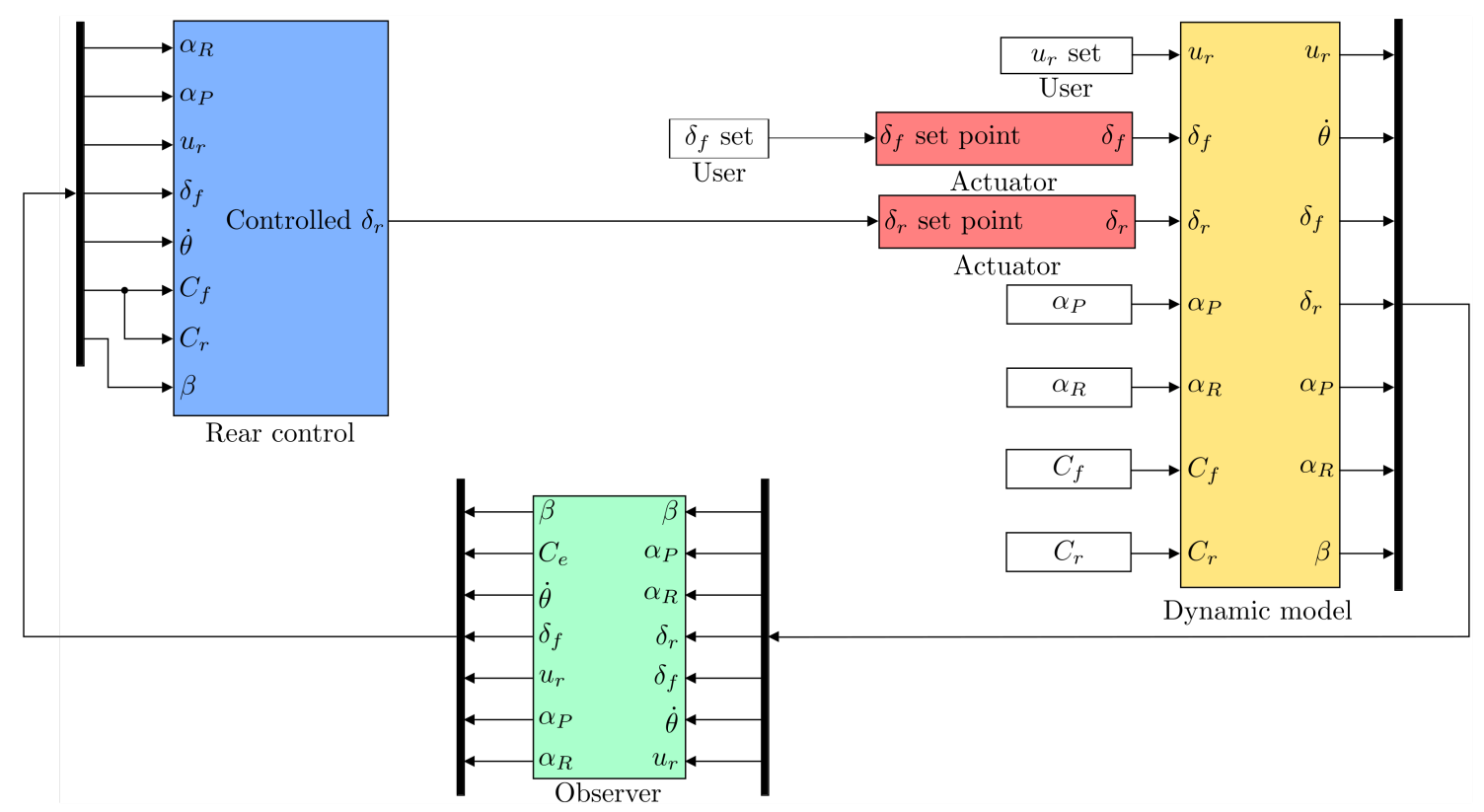

Figure 8. Global simulator scheme

This simulator is composed of different blocks:

- "Actuator" block : In order to represent the actuators as non-ideal, they are considered as second order systems with a $5 \%$ response time of 0.24 seconds.

- "Dynamic model" block : The evolution equations of the dynamic model are implemented here.

- "Rear control" block : The steering law acting on the steering (rear, in this case) gear is implemented here.

- "Observer" block: The cornering stiffness (parameter defining the tyre-ground slip) is reconstructed in this block.

\subsection{Simulation conditions}

Simulations were conducted to test the developed control law and the observer. The vehicle's forward speed is $50 \mathrm{~km} / \mathrm{h}$ and the driver imposes (from 0.5 seconds) a front steering angle of 15 degrees. A sudden change from grippy to slippery ground is simulated at 2.5 seconds (see blue curve in figure 9).

\subsection{Cornering observation results}

The cornering stiffness observation results (in red) presented in Figure 9 are quite good $(0.25$ seconds delay) since the only measurement made to reconstruct the drift stiffness is the yaw rate measurement. 


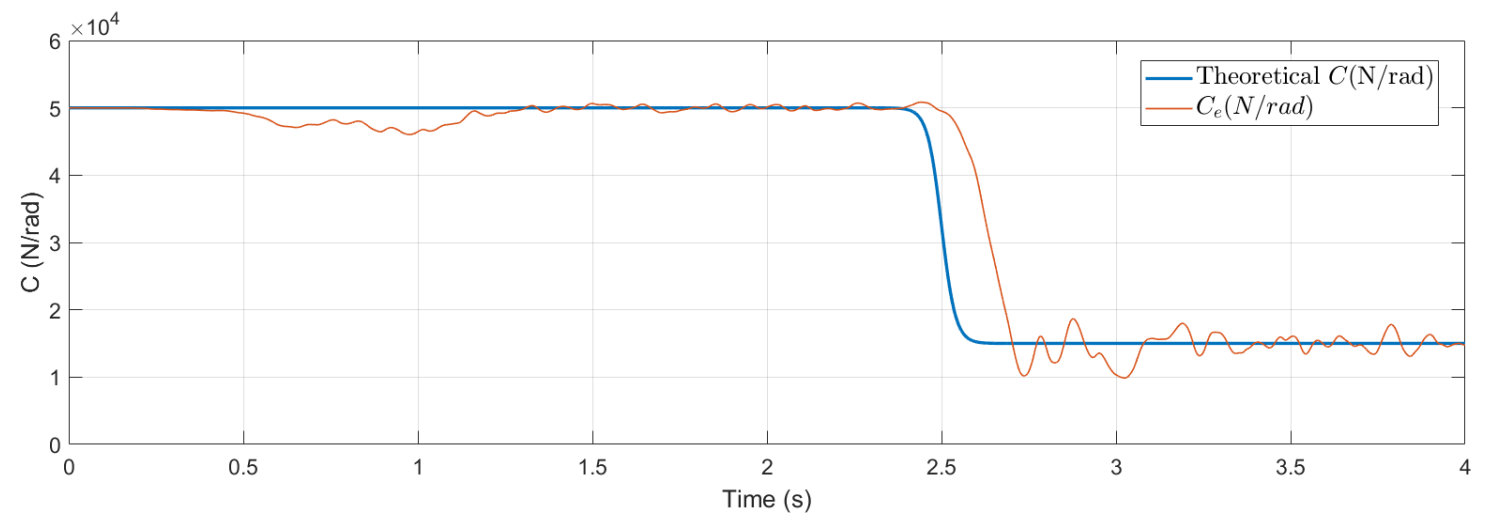

Figure 9. Results of the cornering stiffness observation

\subsection{Steering law results}

As explained in paragraph 5.2, the steering law is tested in a turn, with a sudden change of ground during the turn. According to figure 10, without rear wheel control, the vehicle has an under-steer behaviour: The yaw rate is lower than desired. This under-steer behaviour is accentuated after 2.5 seconds on slippery ground.

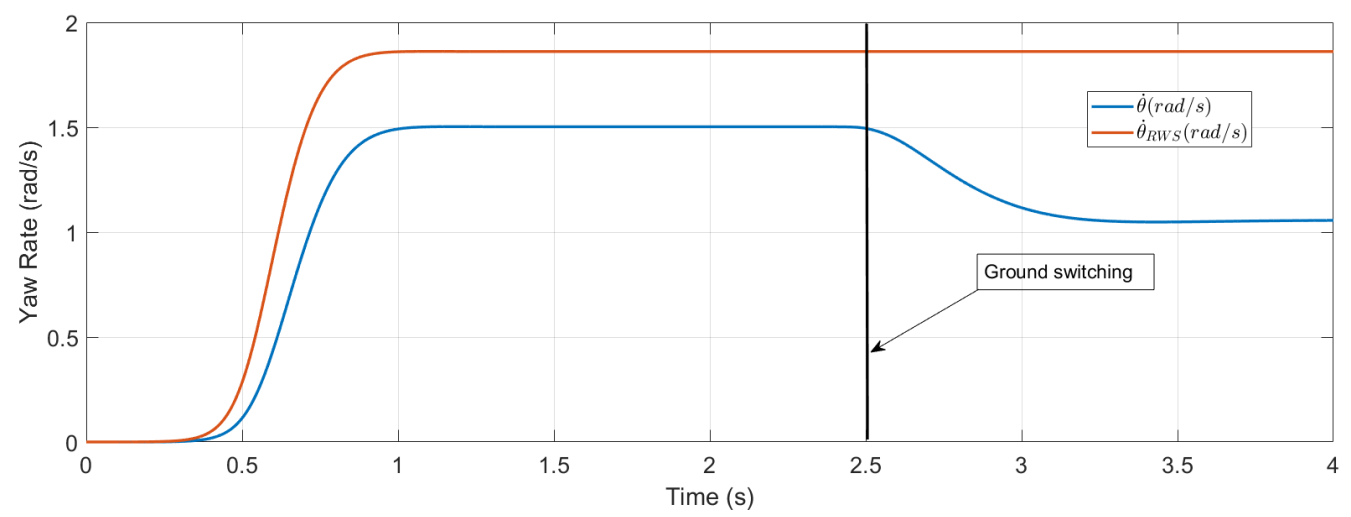

Figure 10. Yaw rate of the vehicle during test

It can be seen on Figure 11 that the control law does exactly what it is asked to do on grippy ground. At 2.5 seconds, the sudden change of ground disrupts the system, but here again the control law make converge the vehicle's yaw rate to its set point.

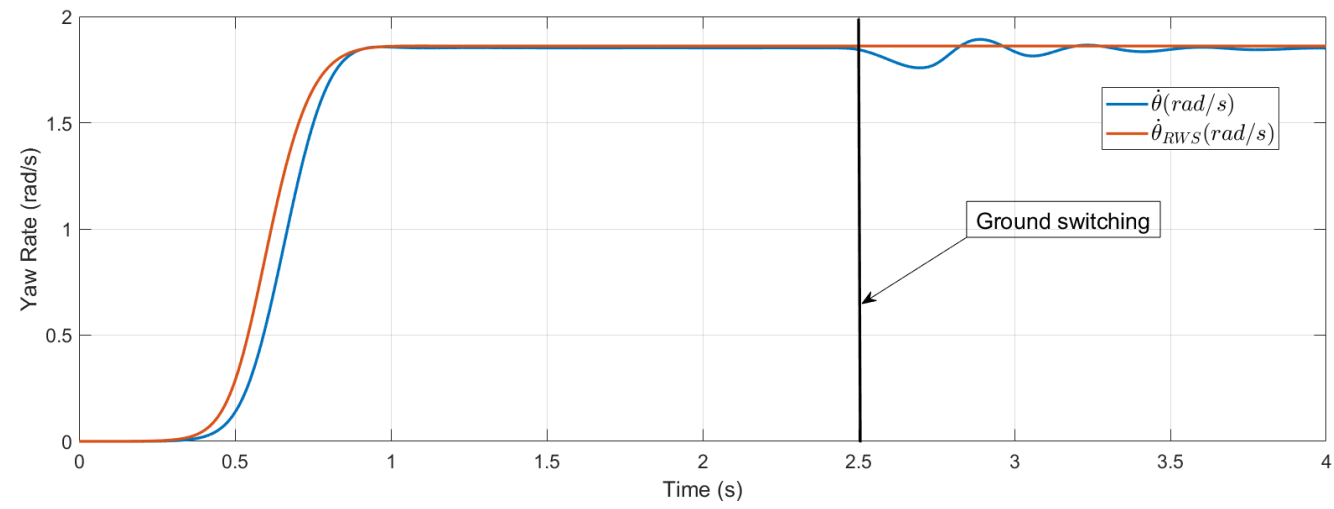

Figure 11. Yaw rate of the vehicle during test 


\section{Conclusion and futur works}

The method presented in this paper fulfils the objective of providing the driver with a driving assistance system that allows him to drive on slippery ground more easily than a vehicle with one steering gear. This objective was achieved by using a control law that ensures the convergence of the vehicle's yaw rate towards a target yaw rate set by the driver. This control law works in parallel with a state observer which, based on an adaptive linear model, reflects the lateral behaviour of the tyre/ground contact.

This has been tested in simulation, with encouraging results for experimental validation on autonomous mobile robots and then on a prototype off-road vehicle (buggy type) with 2 steering gears.

In this paper, only the issue of maintaining a yaw rate has been treated and, more importantly, the control law acts only on one steering gear. By adopting a configuration where the driver does not act directly on one of the steering gears and by allowing the control law to act on both the front and rear gears, the field of possibilities is greatly expanded. Future works will focus on yaw and roll stability issues. This will require new control strategies, in particular optimal and predictive control.

\section{ACKNOWLEDGMENTS}

This work was sponsored by a public grant overseen by the French National Research Agency as part of the "Investissements d'Avenir" through the IMobS3 Laboratory of Excellence (ANR-10LABX-0016) and the IDEX-ISITE initiative CAP 20-25 (ANR-16-IDEX-0001).

\section{REFERENCES}

[1] N. Dal Bianco, R. Lot, and M. Gadola, "Minimum time optimal control simulation of a GP2 race car," Proceedings of the Institution of Mechanical Engineers, Part D: Journal of Automobile Engineering, vol. 232, no. 9, pp. 1180-1195, 2018.

[2] C. Samson, "Control of Chained Systems Application to Path Following and Time-Varying Point-Stabilization of Mobile Robots," IEEE Transactions on Automatic Control, vol. 40, no. 1, pp. 64-77, 1995.

[3] N. Bouton, Stabilité dynamique des véhicules légers tout-terrain. $\mathrm{PhD}$ thesis, 2009.

[4] D. Denis, "Contribution à la modélisation et à la commande de robots mobiles reconfigurables en milieu tout-terrain : application à la stabilité dynamique d'engins agricoles," 2015.

[5] J.-P. Brossard, Dynamique du véhicule Modélisation des systèmes complexes. Presse Polytechniques Romandes, 2013.

[6] R. Lenain, B. Thuilot, C. Cariou, and P. Martinet, "Adaptive and predictive path tracking control for off-road mobile robots," European Journal of Control, vol. 13, no. 4, pp. 419-439, 2007.

[7] C. Cariou, R. Lenain, B. Thuilot, and M. Berducat, "Automatic Guidance of a Four-WheelSteering Mobile Robot for Accurate Field Operations," Journal of Field Robotics, vol. 26, no. 6-7, p. 15, 2009.

[8] E. Bakker, L. Nyborg, and H. B. Pacejka, "Tyre Modelling for Use In Vehicle Dynamics Studies," SAE Technical Papers, vol. 1, 1987.

[9] K. Astrom and B. Wittenmark, Adaptive Control Second Edition. New-York: Addison-Wesley, 1994. 